\title{
IMAGOLOGIA DI FRONTIERA: IL CASO DI TRIESTE
}

Jadranka Cergol, Università del Litorale, Facoltà di studi umanistici, jadranka.cergol@fhs.upr.si

\section{$10.31902 /$ fll. 30.2020 .6}

UDK 82.09(497.12:450.361)

L'articolo si propone di delineare lo stato degli studi imagologici di frontiera analizzando due sistemi letterari che nascono e si sviluppano nello stesso luogo, ma in due lingue diverse: la letteratura italiana e quella slovena in una città che è l'emblema dell'incontro tra le due etnie, cioè Trieste. Dopo un'introduttiva riflessione metodologica sulle letterature comparate e sul metodo imagologico, verranno presi in considerazione soprattutto quegli autori triestini lungo tutto l'arco del $20^{\circ}$ secolo che riflettono nelle loro opere letterarie l'incontro con il vicino, concittadino nella stessa città, ma di lingua e cultura diversa. Saranno messe in risalto alcune considerazioni di tipo storico-letterario che rispecchiano l'ambiente multiculturale di Trieste e il dialogo tra le sue due identità.

Parole chiave: imagologia, Trieste, identità di frontiera, letteratura slovena a Trieste, letteratura triestina, sloveni in Italia

\section{Introduzione metodologica}

Il metodo interdisciplinare dell'imagologia ha come scopo principale quello di intuire le relazioni intrinseche in un gruppo etnico e le sue riflessioni sulla presenza di un altro gruppo etnico e/o linguistico con il quale vive in stretto rapporto. Più che capire la società mira a comprendere il discorso all'interno di una società. II metodo è stato elaborato alcuni decenni fa, si è sviluppato principalmente in Francia grazie a un gruppo di studiosi riuniti intorno al suo fondatore DanielHenri Pageaux ed è incentrato sullo studio delle immagini, dei pregiudizi, delle clichés e degli stereotipi di una determinata cultura vista dalla prospettiva dell'altro. Secondo Pageaux l'applicazione dei metodi imagologici permette agli storici letterari di rivelare le immagini di nazioni diverse, soprattutto nella storia di quei popoli dove una nazione entra in contatto con un'altra (Cergol 213). II metodo imagologico si è dimostrato come un approccio molto fruttuoso per coloro che si occupano di studi dell'altro (sia in campo degli studi 
culturali, sociologici, psicologici, antropologici, storici e letterari) e di come un soggetto riflette l'immagine dell'altro. Nonostante gli scambi culturali facciano già da secoli parte della storia culturale delle singole nazioni, sono stati proprio i cambiamenti sociali degli ultimi anni a dare una spinta maggiore ai ricercatori nel campo dell'imagologia (Beller, Leerssen 2007). L'ambito che offre molte possibilità di analisi è probabilmente proprio la scienza letteraria, nella quale si rispecchiano le "immagini interiori - le immagini della mente e dell'anima" (Scholz 9) che vengono codificate verbalmente e testualmente. Le nostre immagini sono però influenzate da pregiudizi e stereotipi che sono intrinsechi nella nostra cultura e nella nostra percezione ed è per questo che gli studi imagologici ci mostrano molto chiaramente quali sono questi pregiudizi e stereotipi nascosti nelle singole culture. Pageaux propose negli anni ' 80 di esaminare le immagini letterarie di altre nazioni prendendo in considerazione la condizione culturale totale e di aiutarsi con i metodi di vari campi di ricerca umanistica e sociologica. L'immagine delle relazioni tra l'io e l'altro, tra le rappresentazioni interne ed esterne rappresenta un confronto culturale attraverso il quale una persona o un gruppo rivelano il proprio orizzonte ideologico (Pageaux 79-88).

Gli studi imagologici si sono in seguito allargati anche all'ambito tedesco con Franz K. Stanzel e la sua scuola, con Manfred Beller e i suoi studi sulle immagini italo-tedesche, come anche con Nora Moll e Lopez de Abiada's sulle immagini degli spagnoli nelle letterature europee. Ma è di nuovo alla scuola francese che viene attribuito un ulteriore passo metodologico a cura di Jean-Marc Moura che ha proposto una distinzione in tre tipologie di studi: a) "image d'un référent étranger" che prende in considerazione le descrizioni dell'altro come un documento storico b) "image provenant d'une nation ou d'une culture" che prende in considerazione un insieme di attitudini sociali e culturali nell'analisi letteraria e c) "image créé par la sensibilité particulière d'un auteur" che esamina i miti dei singoli autori alla luce di un orizzonte immaginario in un determinato periodo (Moura 184-6; Beller, Leerssen 10). Lo scopo principale degli studi imagologici rimane comunque quello di studiare gli stereotipi culturali e nazionali e non quello di studiare l'identità nazionale o culturale di un gruppo etnico; bisogna quindi sempre parlare delle rappresentazioni come delle strategie testuali. Oltre a ciò bisogna prendere in considerazione che le fonti, essendo queste principalmente fonti letterarie, sono soggettive ed è quindi necessario sempre contestualizzare l'autore e l'opera in un periodo storico preciso e in una società precisa: le opere letterarie non devono essere prese in considerazione nel loro aspetto di opere eterne, 
atemporali. Spesso può altresì succedere che nell'analisi delle immagini degli altri gruppi etnici (hetero-images) vengano messe in risalto anche immagini della propria cultura (self-images).

Usando tutti questi strumenti si cercherà in seguito di presentare gli studi imagologici nel caso di un determinato ambito geografico, cioè quello dell'estremo nord-est italiano, nella città di Trieste che vive in stretto contatto con la cultura slovena, presente sia dall'altra parte del confine italo-sloveno, ma anche e soprattutto nel tessuto stesso della città con una presenza importante della minoranza slovena. Poche sono state le ricerche finora pubblicate che hanno affrontato il tema dell'incontro tra le due etnie e quest'ultime si sono principalmente concentrate sulla situazione letteraria della prima metà del $20^{\circ}$ secolo. Lo scopo del presente contributo è invece quello di affondare le radici anche nel $19^{\circ}$ secolo, quando la componente etnica slovena visse un fiorente periodo di rinascita nazionale e diede vita ad un'importante produzione letteraria che rispecchia in parte anche il confronto e le immagini dei concittadini di origini latine. La ricerca prosegue poi con l'analisi delle immagini tratte dalle opere letterarie lungo tutto il $20^{\circ}$ secolo che sono cambiate in seguito ai cambiamenti politici e sociali occorsi nella città adriatica.

\section{Contesto storico}

La città di Trieste ha vissuto nel $20^{\circ}$ secolo molti cambiamenti politici che hanno fortemente influito sull'immaginario collettivo dell'altro. Nei quattro secoli precedenti la città adriatica ha visto una fiorente crescita economica grazie alla lungimirante politica asburgica che ha reso Trieste porto franco e centro commerciale ed economico dell'impero Austro-Ungarico trasformandola in un'importante città europea multiculturale e multietnica: in essa si incontravano le più moderne correnti culturali, letterarie e sociali - basti pensare agli inizi della psicanalisi che ha potuto mettere le sue radici proprio a Trieste dopo aver subito l'influsso di Vienna. Anche la componente slovena ha vissuto, soprattutto nella seconda metà del $19^{\circ}$ secolo, il suo massimo splendore con tutta una serie di attività culturali ed economiche di alto profilo professionale: teatri, biblioteche, sale di concerto, incontri politici, periodici e riviste letterarie di alto valore etico ed estetico. Dopo questo periodo di crescita economica e culturale, la città è passata dopo la prima guerra mondiale allo Stato italiano, vivendo in quegli anni una crisi d'identità, dato che le sue componenti etniche, sotto le nuove leggi dello stato fascista a partire dal 1922, sono entrate in conflitto tra di loro. Con l'avvento del fascismo e l'introduzione della politica linguistica e razziale del fascismo si è cercato di sradicare la 
componente slovena e in generale quella slava, oltre a quella ebrea, che da secoli era presente sia nell'entroterra triestino che nel tessuto cittadino stesso. I rapporti tra le due anime della città, quella slovena e quella italiana, andavano a deteriorarsi il che è chiaramente evidente anche nelle opere letterarie del periodo. Dopo la seconda guerra mondiale con il Trattato di Parigi nel 1947, Trattato di Londra nel 1954 e il Trattato di Osimo del 1975 Trieste rimase parte integrante della Repubblica italiana. I due gruppi nazionali non riuscirono da subito a superare i conflitti delle due guerre, ma con il passare degli anni, con l'apertura dell'Europa a est e il crescente peso sociale ed economico prima della Jugoslavia, poi della Repubblica di Slovenia anche i rapporti migliorarono. Prova ne è lo scrittore triestino e mitteleuropeo Claudio Magris che dà nelle proprie opere letterarie un'immagine positiva degli sloveni, pur non omettendo gli stereotipi e pregiudizi che spesso persistono nell'immaginario collettivo italiano (Smotlak 2016).

Prendendo in considerazione quindi il periodo letterario del $20^{\circ}$ secolo si nota subito un interesse particolare rivolto alla produzione letteraria triestina in italiano all'inizio del secolo, con autori di fama internazionale come Italo Svevo, Umberto Saba, ai quali vanno aggiunti almeno i fratelli Giani e Carlo Stuparich e Scipio Slataper. Anche dalla parte slovena possono essere citati autori importanti, come Anton Aškerc, Dragotin Kette, Igo Gruden e soprattutto Srečko Kosovel. Ognuno di questi autori è stato largamente analizzato nel proprio sistema letterario, rispettivamente quello italiano e quello sloveno; pochi sono stati invece finora gli studi che hanno preso in considerazione questa produzione come un insieme, analizzando tutti i diversi autori che in realtà provengono dalla stessa città o dal suo entroterra naturale e che scrivono testi letterari rivolti ai propri concittadini. E ancora di meno sono gli studi che analizzano le immagini speculari di uno o dell'altro gruppo etnico.

\section{L'immagine dello sloveno nella letteratura italiana}

\subsection{Prima della prima guerra mondiale}

Nella letteratura triestina scritta in lingua italiana è possibile riscontrare due tendenze principali riguardo l'immagine degli sloveni: un totale occultamento ovvero un'indifferenza di fondo nei confronti della componente slovena (tipica ad esempio per Italo Svevo o Umberto Saba) oppure una valutazione disuguale o addirittura altezzosa nei confronti di coloro che parlavano sloveno (Smotlak 79). Le due tendenze sono riscontrabili anche in periodi diversi: se all'inizio del secolo, prima dello scoppio della guerra, l'atteggiamento era principalmente di indifferenza, questa si trasforma in una leggera 
denigrazione nel periodo tra le due guerre. Tutte queste immagini nacquero e si svilupparono sotto l'influsso di un nazionalismo particolare, tipicamente triestino, noto come irredentismo adriatico. Ma "se I'Irredentismo storico è collocabile tra il 1882 e la fine della prima guerra mondiale, possiamo altresì affermare che la mentalità irredentista sopravvive a Trieste ben al di là dei suoi confini storici e che l'Irredentismo progressivamente assume valenze generalmente antiaustriache e pro-italiane nell'intero arco del XX secolo." (Pizzi 2007, 35).

Un noto esempio di indifferenza ne è quello di Italo Svevo, ma anche Giani Stuparich che spese moltissimo tempo a studiare ad esempio la lingua e la cultura ceca, ignorando completamente i vicini sloveni ovvero presentandoli nelle proprie opere letterarie con delle caratteristiche negative (Thomson 103).

II primo a trattare la componente slovena nella città di Trieste come equivalente a quella italiana fu Angelo Vivante nel suo Irredentismo Adriatico, uscito a Firenze nel 1912, in un clima già molto intriso di nazionalismo italiano, ma ancora incerto sul futuro della città e del suo futuro politico. "Angelo Vivante compone uno dei più imparziali e convincenti saggi a tutt'oggi sulla questione slava" (Pizzi 2007, 145), infatti presenta lo sviluppo culturale, nazionale ed economico della città negli ultimi cento anni e lo riconduce alla sua posizione naturale di importante centro urbano per tutto l'entroterra rurale aggiungendo come la componente slovena e croata debbano avere in questa città gli stessi diritti di quella italiana. Mettendo in risalto la naturale posizione della città e lo sviluppo economico voleva estirpare le radici irredentiste e costruire le fondamenta per una pacifica convivenza tra i diversi gruppi etnici nell'area giuliana. "La convincente analisi si incentra sull'aspetto educativo, dove la lotta di un establishment italiano egemone e purista contro la crescente intellighènzia slava che aspira alla scolarizzazione in lingua slovena risulta più marcata e più aspra" (Vivante 173).

Vivante resta però una delle poche voci italiane che ha dato risalto alla lingua e cultura slovena a Trieste, la maggior parte degli altri autori mostra un atteggiamento di disprezzo e di superiorità che si accentuerà soprattutto dopo l'avvento del fascismo. Leggendo alcuni poeti minori italiani del tempo, si ha l'impressione che in città persistono due gruppi ben distinti da un confine invisibile che divide il "noi" (italiani, protagonisti) e "loro" (sloveni, antagonisti): i primi dimostravano l'italianità di Trieste, i secondi erano i loro antagonisti, ovvero portatori della slovenità di Trieste (Toroš 2019). Un esempio di questa attitudine è possibile riscontrare nella poesia di Edoardo Weiss 'Na volta e adesso: I nostri cari noni / i scazava nazai / a colpi de bastoni 
/ quei prodi del zakai. (...) / In zità i vien urlando: (...) / Ja, zivio Trst slovenska. ${ }^{1 \prime \prime}$ (Toroš 2016, 300). Da questi versi è possibile evincere l'atteggiamento di una netta distinzione tra una componente e l'altra che parlano due lingue diverse attestando che gli sloveni minacciano I'italianità di Trieste. In un crescente clima di irredentismo sempre più marcato gli sloveni venivano infatti visti come una minaccia all'"italianità di Trieste". Nonostante ciò bisogna anche aggiungere che prima dello scoppio della prima guerra mondiale questo sentimento non era prevalentemente espresso con toni molto aggressivi, ma piuttosto ironici e rivolti perlopiù al suono della lingua slovena caratterizzato dal maggiore consonantismo rispetto all'italiano, il che lo dimostra sia la già citata poesia di Weiss come pure i versi di Carlo Mioni: "Perché tentava i 'sciavi / qua farla de paroni / (...) / Za zerti botegheri / meteva le tabele / par crico o in dopia lingua / (...) i libri tavolari / sporcai con pipe e "ici"." (Toroš 2016, 295). La poesia scritta in dialetto indica l'avversione della popolazione italiana alle insegne bilingui sopra le entrate dei negozi, nei quali si parlava anche lo sloveno, come anche alle lettere "con le pipe" š, č, ž, appartenenti all'alfabeto sloveno. "L'ostilità per la minoranza slovena ha radici antiche e, nonostante la coabitazione prolungata di diverse etnie, prevale la tendenza a considerare la comunità slovena come straniera e intrusiva: nella dialettica "insider" vs. "outsider" (...) Una paventata, leggendaria "calata" degli slavi dalle montagne verso il cuore della civiltà latina abbonda di similitudini grottesche e truculente, come si legge in Giulio Caprin e Desico (Enrico Schott), e come si evince, sia pur smorzato nei toni, velato di affettuoso paternalismo o esulcerato in uno spettacolo di rapacità omicida, anche in poeti importanti quali Giotti e Marin" (Pizzi 2016, 146).

Nella letteratura e nella saggistica triestina di lingua italiana, pubblicata prima dello scoppio della prima guerra mondiale si possono quindi riscontrare diversi atteggiamenti, nati soprattutto sotto l'influsso del prevalente clima irredentista della città: da una parte si trovano gli intellettuali che auspicano una pacifica convivenza tra i gruppi etnici (ad es. Vivante) e dall'altra autori che ignorano completamente l'esistenza di questa componente della città. Ancora un terzo gruppo di autori dà invece sfogo alla tensione nazionale che si era andata a creare nei decenni precedenti e che vedeva negli sloveni una minaccia all'italianità della città. "In questo contesto l'Altro non veniva visto come vicino, ma come straniero incolto e barbaro minaccioso" (Toroš 2011, 96).

1 “Sì, evviva la Trieste slovena”. 


\subsection{Tra le due guerre}

S'ciavo, vuoi venire con me? lo ti faccio padrone delle grandi campagne sul mare. Lontana è la nostra pianura, ma il mare è ricco e bello. E tu devi esserne padrone. Perché tu sei slavo, figliolo della nuova razza (Slataper 37).

Scipio Slataper fu uno dei primi autori di lingua italiana a prendere in considerazione la componente slovena della città e del suo entroterra. II passo appena citato mostra però un rapporto estremamente emblematico che gli intellettuali italiani di Trieste avevano nei confronti del mondo sloveno già prima dell'avvento del fascismo. Questo rapporto problematico può essere notato sempre nello stesso Slataper che da una parte cantava la pacifica convivenza tra i due popoli, la plurietnicità della città e la sua collocazione strategica all'interno dell'Impero asburgico, dall'altra invece voleva rintanare il popolo sloveno sul Carso, nelle campagne, senza permettergli di prendere in mano il dominio politico della città (Cergol 235), come si può evincere dai passi:

bisogna permettere tutte le manifestazioni colturali slave e tedesche, magari favorendole. Ma impedire fin dal primo giorno con decisa serietà, ogni moto politico (Slataper 40).

E ancora:

L'urbanesimo pertanto avrebbe dovuto essere prevalentemente italiano (veneto-friulano, romagnolo, pugliese soprattutto) e la borghesia slava dovrà dileguarsi a poco a poco, non per costrizione legale ma perché i posti governativi e in parte comunali non saranno più soltanto per lei, e i medici e gli avvocati slavi avranno sempre meno da fare, mancando via via i clienti della Carinzia e della Carniola. I pochi agricoltori slavi che resteranno sarà facile sorvegliarli e tenerli a freno (idem).

Dalle parole di Scipio Slataper è quindi evidente il rapporto degli italiani nei confronti degli sloveni che venivano considerati come principalmente appartenenti al mondo rurale, dall'altra invece la borghesia slovena (avvocati, medici ecc.) non era ben vista dai concittadini di lingua italiana che volevano ottenere il dominio politico e sociale della città.

Da questo fattore sociologico scaturisce infatti un rapporto degli italiani nei confronti degli sloveni di tipo protezionistico e appunto alquanto altezzoso, dato che gli sloveni vengono considerati dai più gente prevalentemente contadina senza cultura e prestigio storico.

Un atteggiamento invece più di disprezzo è possibile notare nel romanzo di Giani Stuparich Ritorneranno, dove troviamo Berta - 
"l'ispida servetta di casa, una slovena dell'alto Isonzo" (Senardi 372), ma anche una "bestia selvatica che si lascia difficilmente avvicinare" (Stuparich 17). Un atteggiamento simile può essere notato anche in Pier Antonio Quarantotti Gambini (Tremul 2008) che attribuisce agli sloveni addirittura una fisionomia diversa da quella degli italiani:

Questi sloveni della campagna (...): uniformemente bassi e ossuti, biondicci e scabri - sembrano non cresciuti qui vicino ma di tutt'altri paesi, a paragone dei triestini che sono alti e biondi (Quarantotti Gambini 125).

\subsection{Nel secondo dopoguerra}

“È possibile notare un'apertura maggiore e più sensibile nei confronti degli sloveni dagli anni '60 in poi. Soprattutto Fulvio Tomizza nelle proprie opere descriveva gli sloveni attraverso dei personaggi che erano alla pari degli italiani" (Smotlak 79). Mentre Fulvio Tomizza è considerato lo scrittore di frontiera par excellence, dato che è stato colui che ha saputo maggiormente esprimere nelle proprie opere le tragedie personali e collettive, le crisi, la difficile convivenza tra le due etnie dopo la seconda guerra mondiale, ma è stato anche finora l'unico scrittore italiano a trattare in un suo romanzo, Gli sposi di via Rossetti, una vicenda del tutto "slovena", presentando la sorte di alcuni intellettuali sloveni soprattutto da un punto di vista umano, senza pregiudizi, né stereotipi nei confronti delle varie parti che compongono una storia che è ancora oggi intrisa di mistero politico.

Ma oltre Tomizza è possibile citare anche un autore meno conosciuto, Bruno Steffè, e il suo romanzo I cavalli di guerra non amano la pace, uscito nel 1964, in cui si raccontano gli anni difficili del dopoguerra a Trieste, cercando di fare chiarezza sulle vicende individuali e collettive di una città divisa dai confini e dalle ideologie contrapposte. II romanzo racconta infatti di un ex-partigiano italiano, Aldo, che ha combattuto a fianco dei partigiani sloveni durante la seconda guerra mondiale, ma che al suo ritorno si ritrova circondato da una borghesia italiana anti-slava. II romanzo si chiude comunque con I'idea di una riconciliazione e coabitazione pacifica dei diversi gruppi etnici, come viene spiegato alla fine: "I'atmosfera nazionalistica si è svelenita. La gente ha ripreso a considerare con realtà la situazione e pensa a vivere (...). Sarebbe ora che questa città finisse di essere 'estremo baluardo' per diventare piuttosto un punto d'incontro dei due mondi" (Steffé 402). Anche i suoi successivi romanzi, usciti negli anni '90 raccontano delle due anime della città, in conflitto tra loro, eppure imparentate. 
Tra gli autori che si sono maggiormente aperti nei confronti non soltanto della componente slovena, ma anche delle altre componenti etniche che formano il tessuto della città di Trieste, c'è Claudio Magris, autore poliedrico, considerato anche come scrittore dall'"identità plurale" (Pellegrini 3-10), ma anche dell'eterogeneità e della transizione (Smotlak 81). Nel saggio "Podoba Slovencev v leposlovnem opusu Claudia Magrisa" ("L'immagine degli Sloveni nell'opera letteraria di Claudio Magris") viene chiaramente messo in risalto come nessuno dei protagonisti di Magris non sia sloveno di origini, la comunità viene percepita come un'entità omogenea e astratta che la considera come legata a delle categorie del passato alle quali difficilmente recedono. Una di queste categorie è il mito della Jugoslavia, mentre rimane molto viva anche la memoria storica, legata soprattutto alla prima metà del $20^{\circ}$ secolo, ovvero al periodo della seconda guerra mondiale. Magris è soprattutto interessato ai temi della resistenza partigiana, al simbolismo del $1^{\circ}$ maggio 1945 e alla cessione da parte dell'Italia dell'Istria, di Fiume e della Dalmazia. Nelle sue opere cerca di essere imparziale, dato che presenta anche il lato più buio del fascismo triestino e della violenza sulla popolazione slovena. Magris approfondisce anche le radici dell'attrito italo-sloveno e cerca di superarlo. Magris comunque non nasconde, secondo la Smotlak, alcuni stereotipi che affondano le proprie radici nell'irredentismo triestino come ad esempio l'errata sinonimia tra i termini "Sloveni" e "Slavi" oppure il legame degli sloveni con il mito della Jugoslavia che mostrano come persistono ancora alcune caratteristiche della mentalità altezzosa della superiorità italiana nei confronti della componente slovena della città.

\section{L'immagine dell'italiano nella letteratura slovena del $20^{\circ}$ secolo}

Trattando dell'immagine degli italiani nella letteratura slovena bisogna subito all'inizio precisare che pochi sono gli studi condotti in questo campo; nonostante ciò, tenteremo di delineare alcune caratteristiche comuni a più autori nei confronti della nazione vicina. Anche in quest'ottica bisogna aggiungere che sono pochi gli autori che mostrano un'immagine del vicino: mentre la letteratura slovena è intrisa di stereotipi nei confronti dei vicini meridionali, sembra che quelli occidentali non destino molto interesse, tranne negli autori che erano in stretto contatto con il popolo italiano. "La poesia slovena non ha riflesso fino alla fine della prima guerra mondiale gli attriti nazionali nella città di Trieste e neanche nessuna problematicità linguistica. Prevalentemente ha preso in prestito alcune parole straniere 
dall'italiano, quando ha voluto mostrare la vivacità e il fervore della città" (Toroš 2011, 65). La stessa ricercatrice Ana Toroš che finora ha maggiormente studiato questi contatti afferma che $i$ poeti sloveni hanno cominciato a riflettere le proprie esperienze di Trieste e degli italiani appena dopo la prima guerra mondiale quando Trieste ha cominciato a far parte del Regno d'Italia e quando è cominciata una violenta italianizzazione della popolazione slovena in queste terre (Toroš 2016, 7).

Nonostante ciò alcune immagini degli italiani prima dello scoppio della prima guerra mondiale sono riscontrabili già prima.

\subsection{Prima della prima guerra mondiale}

Le immagini degli italiani nella letteratura slovena sono riscontrabili già nella seconda metà del $19^{\circ}$ secolo, quando l'italiano viene presentato come un personaggio che parla velocemente e mentre parla gesticola vivacemente. Spesso agli italiani veniva attribuita una certa ignoranza o addirittura maleducazione. Negli scritti sulle guerre e sulle battaglie gli italiani vengono presentati come codardi e anche come una nazione che si mette sempre dalla parte del più forte. Un esempio della figura di un italiano è la figura di Birbantino nel romanzo Za svobodo in ljubezen (Per la libertà e l'amore) di Fran Levstik, che viene presentato come un personaggio dalla carnagione alquanto scura e bramoso di guadagni immediati. Le immagini degli italiani nascevano soprattutto sui vari fronti di guerra: in questo contesto vengono messi in risalto soprattutto la timidezza e l'incapacità dei soldati italiani a combattere valorosamente, come descritto dallo scrittore Janez Trdina:

Cucinavano da soli e mangiavano orrendamente una polenta piena di ogni schifezza; bestemmiavano come carrettieri sulle grandi vie. (...) E si gettavano sulle donne come il lupo sugli agnelli, molte bruttezze si raccontavano su di loro a tal riguardo (Trdina 40).

La situazione a Trieste era alquanto diversa, dato che i contatti tra le due etnie erano prevalentemente di natura commerciale. Per questo motivo gli sloveni non avevano un atteggiamento eccessivamente negativo verso la cultura italiana. Gli autori sloveni di Trieste si soffermavano di più sugli aspetti sociali degli agricoltori sloveni o sugli aspetti intimistici della poesia, come nella raccolta Jadranski biseri (Le perle dell'Adriatico) di Anton Aškerc o nella poesia di Dragotin Kette Na molu San Carlo (Sul molo San Carlo).

È possibile però riscontrare a volte anche un atteggiamento di rifiuto nei confronti dei competitori sul mare, i chioggiotti che si 
disputavano i pesci nell'Adriatico settentrionale con i pescatori sloveni triestini. Nella Balada o Čožotih (Ballata sui Chioggiotti) di Anton Aškerc è possibile riscontrare degli attriti tra le due etnie e un atteggiamento di rifiuto da parte degli sloveni nei confronti degli italiani, dovuto soprattutto ad una reazione negativa quando veniva loro attribuito l'epiteto di "sciavi". Aškerc, infatti, scrive: "Čožoti, / kazali nam osle, / pitali nas s 'ščavi', / kadar so veslali / drzni mimo nas" (I chioggiotti ci mostravano dei brutti gesti, ci gridavano 'ščavi' quando con atteggiamento altezzoso remavano davanti a noi). La parola 'ščavo' è spesso presente nelle poesie italiane in dialetto prima della prima guerra mondiale e indica un atteggiamento di disprezzo nei confronti della popolazione slovena; l'imprecazione veniva usata insieme ad altre forme di caricature e di denigrazione degli sloveni che venivano così considerati una popolazione che deve essere sottomessa a quella italiana. Questo atteggiamento negativo nei confronti degli sloveni deriverebbe secondo Magris e Ara da un sentimento di minaccia nel vedere la crescita demografica, culturale ed economica della componente slava in città (Ara, Magris 68).

\subsection{Tra le due guerre}

Con l'avvento del fascismo e delle leggi che avevano lo scopo di sradicare la componente alloglotta della città le immagini dell'altro si sono intensificate. Infatti gli stereotipi negativi, come è stato dimostrato dagli studi comparativisti, si intensificano in concomitanza con i conflitti bellici o anche con attriti politici. La maggior parte delle poesie parla dell'Altro (italiano) che è più forte e minaccia la sua esistenza politica, economica e culturale. In quest'ottica la salvaguardia della lingua slovena è diventata anche la salvaguardia dell'identità nazionale, ma soprattutto dell'integrità umana (Toroš 2011, 66). Gli autori sloveni, nati nelle zone del Litorale e che hanno subito la politica di assimilazione forzata, hanno creato l'immagine dell'italiano coincidente a quella del fascista crudele, che vuole sradicare la nazione slovena dalla propria terra (Cergol 237). Sulla base teoretica tutto ciò è possibile intendere come un processo naturale di un popolo che vuole distanziarsi dall'immagine attribuitagli dal popolo accanto (Moll 1995).

Le immagini dell'italiano sono nella letteratura slovena prevalentemente negative, l'italiano viene considerato come uno straniero, come "il discendente di Nerone" che ha portato nella città solo distruzione incendiando il centro della vita slovena, il Narodni dom, proprio così come aveva incendiato la sua città l'imperatore romano (la poetessa dallo pseudonimo Rodoljubka, Kobletnici 13. julija - Nell'anniversario del 13 luglio). A prevalere è quindi un senso di 
impotenza davanti alla violenza distruttrice del regime fascista, infatti gli sloveni, essendo politicamente più deboli, non potevano opporsi all'oppressione e riflettono la loro situazione caratterizzando l'altro con gli epiteti di banda nera (črna tolpa), usato da Ljubka Šorli, estranei sinistri nell'era buia (mrki tujci v temačni dobi), come vengono invece definiti da Igo Gruden.

\section{Conclusioni}

Da questa presentazione degli studi imagologici di frontiera sul caso della città di Trieste è possibile trarne alcune conclusioni. Dopo che la città aveva raggiunto nel periodo austro-ungarico la sua massima espansione sia economica che culturale avendo accolto tra le proprie vie e palazzi gruppi etnici che arrivavano da tutto il mediterraneo, ha vissuto un periodo stagnante nel $20^{\circ}$ secolo, soprattutto tra le due guerre. Se da una parte prima dello scoppio della prima guerra mondiale è potuta diventare crogiuolo di culture, tra le quali spiccavano soprattutto la cultura italiana da una parte e le varie culture e lingue slave dall'altra, l'irredentismo adriatico e le seguenti vicende storiche hanno causato delle barriere tra le comunità esistenti nella città. Le immagini che ogni singolo gruppo si è formato dell'Altro erano all'inizio stereotipi molto comuni alle singole popolazioni (italiani che gesticolano mentre parlano; lingua slovena molto gutturale con simboli strani), ma in realtà non mostravano un particolare attrito ed erano limitate ad un atteggiamento ironico nei confronti del vicino. Le immagini si trasformarono nel primo dopoguerra con l'avvento del fascismo e delle leggi che volevano sradicare le popolazioni alloglotte della città. Con il passare degli anni l'immagine dell'italiano ha cominciato ad essere identificata con l'immagine del fascista, vestito di nero, che vuole dominare sulla popolazione slovena che si presenta come vittima della situazione socio-politica. I rari studi eseguiti sulle opere letterarie del secondo dopoguerra hanno dimostrato che queste immagini vanno pian piano sfumando: il concittadino sta cominciando ad essere accettato come parte integrante del tessuto urbano ed extraurbano. Bisogna però aggiungere che persistono ancora in certi scritti immagini che sono state forgiate nel primo dopoguerra, il che ci fa pensare che Trieste non abbia saputo approfittare della sua posizione geografica e culturale diventando un esempio di città transculturale o crocevia di culture. Viene invece ancora percepita come un "baluardo" dell'italianità, il che rappresenta un ostacolo per il suo sviluppo economico e culturale. "Confusione e confronto sono più potenti, addirittura fanatici nelle zone di confine. Una regione che era stata storicamente insicura di sé, e una città, Trieste, in perenne ricerca di 
una sua identità letteraria, può diventare molto vulnerabile alle minacce di una chiusa, non confrontabile identità mono-culturale. Questa ricerca perenne di una mono-cultura è di nuovo molto tipica per le zone di confine, dove l'identità viene vista in una luce negativa, in opposizione alla cultura e alla lingua dell'Altro." (Pizzi 2016, 123).

In conclusione potremmo auspicare a questa regione come pure ad altre regioni di frontiera soprattutto una diversa visione della propria identità alla luce delle metodologie di ricerca degli studi comparativisti. Alcuni autori hanno mostrato con la propria identità culturale, il proprio impegno politico, con il proprio respiro europeo ed internazionale la via per costruire ponti che possono collegare i vari gruppi etnici nella stessa città che la storia ha purtroppo diviso.

\section{Bibliografia:}

Ara, Angelo and Claudio Magris. Trieste: un'identità di frontiera. Torino: Einaudi, 2001.

Beller, Manfred, and Joep Leerssen. Imagology: The cultural construction and literary representation of national characters. Amsterdam-New York: Rodopi, 2007.

Cergol, Jadranka. "L'immagine dello sloveno nella letteratura triestina italiana e l'immagine dell'italiano nella letteratura slovena nel periodo fascista: confronto tra due stereotipi." Literature in an Intercultural Perspective. Ed. Nives Zudič Antonič. KoperVenezia, Annales University Press-Dipartimento di Studi Linguistici e Culturali Comparati, 2015. 231-240.

Moll, Nora. "L'immagine dell'altro. Imagologia e studi interculturali." L'immagine dell'altro e l'identità nazionale: metodi di ricerca letteraria. Ed. Manfred Beller. Fasano: Schena, 1996. 221-48.

Moura, Jean-Marc. "L'imagologie littéraire: tendances actuelles." Perspectives comparatistes. Ed. di Jean Bessière and DanielHenri Pageaux. Paris: Champion, 1999. 184-6.

Pageaux, Daniel-Henri. "Une perspective d'études en literature compare à l'antropologie culturelle." Synthesis 10 (1981): 7988.

Pellegrini, Ezio. "Introduzione." Claudio Magris: Opere. Ed. Ezio Pellegrini. Milano: Einaudi, 2016. 3-10.

Pizzi, Katia. Trieste: italianità, triestinità e male di frontiera. Bologna: Gedit, 2007.

Cold war cities: history, culture and memory. Oxford: P. Lang, 2016. 
Quarantotti Gambini, Pier Antonio. Primavera a Trieste e altri scritti, Trieste: Edizioni Italo Svevo Dedolibri, 1985.

Scholz, Oliver R. Bild, Darstellung, Zeichen: Philosophische Theorien bildhafter Darstellung, Frankfurt: KlostermannRoteReihe, 2004.

Senardi, Fulvio. "Giani Stuparich, testimone e narratore della Grande Guerra." Trento e Trieste: percorsi degli italiani d'Austria dal '48 all'annessione: atti del convegno Rovereto 1, 2, 3 dicembre 2011: in onore di Livio Caffieri. Ed. Fabrizio Rasera and Livio Caffieri; Rovereto: Accademia Roveretana degli Agiati, Osiride, 2014. 361-375.

Slataper, Scipio. I/ Mio Carso. Firenze: Libreria della voce, 1912.

------- Lettere. Torino: Fratelli Buratti, 1931.

Smotlak, Maja. "Podoba Slovencev v leposlovnem opusu italijanskega tržaškega pisatelja Claudia Magrisa." Primerjalna književnost, 34.3 (2016): 77-96.

Steffè, Bruno. I cavalli di guerra non amano la pace. Trieste: Bruno Steffè, 1964.

Stuparich, Giani. Ritorneranno, Milano: Garzanti, 1991.

Thomson, Mark. The White War: Life and Death on the Italian Front 1915-1919. London: Faber and Faber, 2008.

Toroš, Ana. Podoba Trsta in Tržaškega v slovenski in italijanski poeziji prve polovice 20. stoletja, Nova Gorica: Univerza v Novi Gorici, 2011.

Auto-stereotypes and Hetero-stereotypes in Slovene and Italian Poetry About Trieste From the First Half of the 20th Century. Interlitteraria 21/2 (2016): 290-304.

Potovanje po neznanih poteh tržaškosti in Gradnikove poezije: izbrani spisi, Trieste: Mladika, 2019.

Trdina, Janez. Moje življenje. Ljubljana: Državna založba Slovenije, 1947.

Tremul, Maurizio. Pier Antonio Quarantotti Gambini: atti del convegno di studi. Capodistria: Unione italiana, 2008.

Vivante, Angelo. Irredentismo adriatico. Firenze: Parenti, 1912.

\section{GRANIČNA IMAGOLOGIJA: SLUČAJ TRSTA}

Cilj članka je da prikaže status pograničnih imagoloških studija analizirajući dva književna sistema koji su nastali i razvili se na istom mjestu, ali na dva različita jezika: italijanska i slovenačka književnost u gradu koji je simbol susreta dvije nacije, to jest Trstu. Nakon uvodnog metodološkog komentara o komparativnoj književnosti i imagološkom metodu, u razmatranje će biti uzeti posebno autori iz 20. vijeka koji u 
svojim književnim djelima opisuju susret s komšijom, stanovnikom istog grada, ali različitog jezika i kulture. Prije svega će biti istaknuta razmatranja, kako istorijskog tako književnog tipa, koja odražavaju multikulturalnu sredinu Trsta i dijalog između njegova dva identiteta.

Ključne riječi: imagologija, Trst, granični identitet, slovenačka književnost u Trstu, tršćanska književnost, Slovenci u Italiji 